\title{
Global Transcriptome Analysis of Cucmber (Cucumis Sativus L.) in Response to Cucurbit Chlorotic Yellows Virus
}

\author{
Hsiu-fung Chao', Yung-fu Yen ${ }^{2}$ \\ ${ }^{1}$ Tainan District Agricultural Research and Extension Station \\ 70 Muchang, Hsinhua District, Tainan, Taiwan \\ hfchao@mail.tndais.gov.tw \\ ${ }^{2}$ Department of Bioagricultural Science, National Chiayi University \\ 300 University Road, Chiayi, Taiwan \\ yfyen@mail.ncyu.edu.tw
}

\section{Extended Abstract}

The Cucurbit chlorotic yellows virus (CCYV) may infect many crops of Cucurbitaceae that leaves appear chlorosis or chlorotic spots and fruits yield low and are poor quality. Lately, the epidemic virus of CCYV has severely out-broken in field/greenhouse-grown cucurbits, and caused enormous losses of cucurbit industry. CCYV disease was first identified in Japan in 2004 and has rapidly spread in Asian, now CCYV has become to one of the most important epidemic virus of cucurbit crops. Until now there do not resistant cultivars released due to resistant gene of CCYV of cucurbits is not found. Recently resistant/tolerant plants of cucumber to CCYV have been identified by molecular diagnosis in my Lab. For identifying genes which involve CCYV resistance, we surveyed whole transcripts of cucumber (Cucumis sativus L.) inbred line 'Feng-tyan no.6', a tolerant line, through next generation sequencing (NGS). Total RNAs were extracted and whole transcriptome shotgun sequencing was performed using an Illumina platform. The gene expression level was calculated as FPKM. For differential expression analysis, CummeRbund was employed to perform statistical analyses of gene expression profiles. NGS detected a total of 19 differentially expressed genes (DEGs) with a higher abundance (>1-fold change with a $\mathrm{p}$ value <0.001) in the CCYV resistant transcriptome compared to that of the CCYV susceptible transcriptome. Among these DEGs relate to metabolic pathways, signalling pathways, DNA replication and cell cycle. The expression of these DEGs was further corroborated by PCR results. A comprehensive analysis of their gene expression profiles revealed that 3 genes (Gene ID: 101207198, 105435998 and 105436080) analysed were consistently resistance regulated by RT-PCR, indicating that the responses induced by CCYV. Moreover, the gene expression levels of 3 resistance regulated genes of 'Her torng no.11' of CCYV tolerant cultivars of cucumber was investigated at early and late stages of plants by real-time PCR, the results showed that the expression levels of 3 genes (Gene ID: 101207198, 105435998 and 105436080) of healthy plant are 400-826 folds, and are only 0.5-8.2 folds of disaster plants. The studies have provided the first glimpse of the molecular breeding of cucumber to resistant CCY disease. 